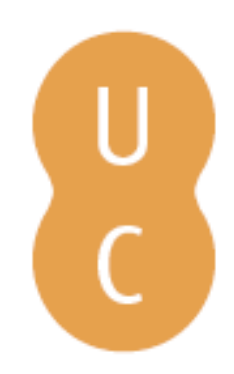

\title{
nommalina
}

\section{Modelação de processos de queda de blocos em vertentes na região de Alvaiázere: dois estudos caso}

\author{
Autor(es): $\quad$ Santos, Pedro; Tavares, Alexandre \\ Publicado por: Imprensa da Universidade de Coimbra; Laboratório de Radioactividade \\ Natural da Universidade de Coimbra \\ URL $\quad$ URI:http://hdl.handle.net/10316.2/36316 \\ DOI: $\quad$ DOI:http://dx.doi.org/10.14195/978-989-26-1009-2_27 \\ Accessed : $\quad$ 26-Apr-2023 16:15:16
}

A navegação consulta e descarregamento dos títulos inseridos nas Bibliotecas Digitais UC Digitalis, UC Pombalina e UC Impactum, pressupõem a aceitação plena e sem reservas dos Termos e Condições de Uso destas Bibliotecas Digitais, disponíveis em https://digitalis.uc.pt/pt-pt/termos.

Conforme exposto nos referidos Termos e Condições de Uso, o descarregamento de títulos de acesso restrito requer uma licença válida de autorização devendo o utilizador aceder ao(s) documento(s) a partir de um endereço de IP da instituição detentora da supramencionada licença.

Ao utilizador é apenas permitido o descarregamento para uso pessoal, pelo que o emprego do(s) título(s) descarregado(s) para outro fim, designadamente comercial, carece de autorização do respetivo autor ou editor da obra.

Na medida em que todas as obras da UC Digitalis se encontram protegidas pelo Código do Direito de Autor e Direitos Conexos e demais legislação aplicável, toda a cópia, parcial ou total, deste documento, nos casos em que é legalmente admitida, deverá conter ou fazer-se acompanhar por este aviso.

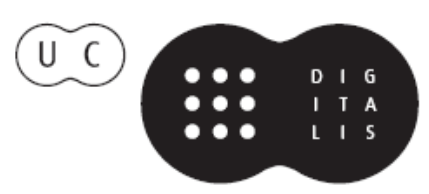




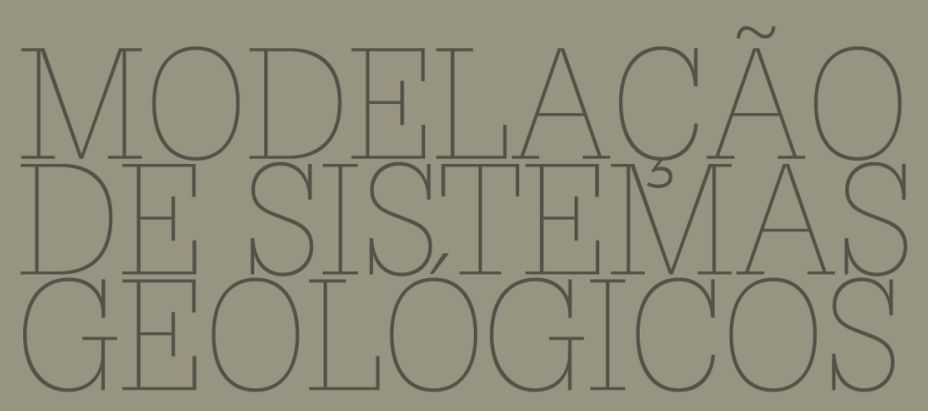

Homenagem ao Professor Doutor Manuel Maria Godinho

L.J.P.F. Neves, A.J.S.C. Pereira,

C.S.R. Gomes, L.C.G. Pereira,

A.O. TAVARES

IMPRENSA DA UNIVERSIDADE DE COIMBRA

CoImBra University Press 


\title{
MODELAÇÃO \\ DE SISTEMAS \\ GEOLÓGICOS
}

Homenagem ao Professor Manuel Maria Godinho

\section{Modelação de processos de queda de blocos em vertentes na região de Alvaiázere; dois estudos caso}

\author{
Pedro Santos $^{1}$; Alexandre Tavares ${ }^{2}$ \\ ${ }^{1}$ Departamento de Ciências da Terra - FCTUC, PORTUGAL, psantos@dct.uc.pt \\ ${ }^{2}$ Departamento de Ciências da Terra - FCTUC e Centro de Estudos Sociais da Universidade de \\ Coimbra, PORTUGAL, atavares@ci.uc.pt
}

Palavras-chave: Queda de blocos, RockFall Analyst, Susceptibilidade, Alvaiázere

\section{Resumo}

A região de Alvaiázere apresenta características geológicas e geomorfológicas muito contrastadas que resultam do contacto entre terrenos da Orla Mesocenozóica e do Maciço Hespérico, fortemente deformados por acção tectónica antiga e recente. Este enquadramento tem potencialmente um efeito condicionante sobre os processos de movimentos de massa em vertente. $\mathrm{O}$ estudo que se apresenta descreve a aplicação de um método de modelação tri-dimensional do processo de queda de blocos na região de Alvaiázere, realizado de modo a caracterizar a susceptibilidade de ocorrência. A cartografia de susceptibilidade foi obtida recorrendo à extensão RockFall Analyst, que corre sobre a plataforma ArcGIS. A aplicação do modelo realizou-se em dois contextos genericamente distintos - um referente a terrenos do Maciço Antigo e outro em terrenos jurássicos que incluem a Serra de Alvaiázere e a sua envolvente. Nestes locais, seleccionaram-se como "áreas de alimentação" (seeders) aquelas que apresentavam declive superior a $20^{\circ}$. Tendo em conta os factores condicionantes dos processos, introduzidos como dados de entrada no modelo - factores morfológicos, litológicos e de ocupação do solo - os resultados obtidos parecem evidenciar uma preponderância do factor declive como o principal condicionante da distribuição das classes de susceptibilidade. É ainda de salientar uma preponderância de movimentos entre $15 \mathrm{~m}$ a $30 \mathrm{~m}$ na distancia de projecção.

Key-words: Rock fall, RockFall Analyst, Susceptibility, Alvaiázere

\begin{abstract}
The region of Alvaiázere presents contrasted geological and geomorphological characteristics, that comes from representation of two morpho-structural domains, the Mesocenozoic Basin and the Hesperian Massif, deformed by ancient and recent tectonic activity. This framework have potential conditioning effect on the processes of slope mass movements. The study describes the application of three-dimensional modeling of the process of rock fall in the region of Alvaiázere, carried out to characterize their susceptibility of occurrence. The mapping of susceptibility was obtained using the RockFall
\end{abstract}


Analyst extension, which runs on the ArcGIS platform. The application of the model took place in two distinct areas - one located in the grounds of the Hesperian Massif, northeastern sector of Alvaiázere municipality, and the other in the Jurassic domains including Alvaiázere mountain range and its surrounding areas. In these two case studies, seeder areas were selected where slope exceeded 20 degrees. Given the conditioning factors considered and introduced as input data in the model - morphological, lithological and land use - the results seem to indicate a preponderance of the slope factor as the main determinant of susceptibility classes distribution. The analysis also demonstrated that mostly the average projection distance has range values between $15 \mathrm{~m}$ and $30 \mathrm{~m}$.

\section{Introdução}

\section{Âmbito e objectivos}

O estudo realizado inseriu-se no âmbito de um acordo-programa estabelecido entre o Departamento de Ciências da Terra da Faculdade de Ciências e Tecnologia da Universidade de Coimbra e o Município de Alvaiázere, ao abrigo do qual se têm produzido uma série de elementos relativos à gestão do risco, incluindo cartografia de susceptibilidade a movimentos de massa em vertente.

Em função das características biogeofísicas e de ocupação humana do território, a queda de blocos foi identificada como um dos processos a caracterizar. Consideram-se abrangidos no conceito de queda de blocos os processos de movimento, por desprendimento, de materiais rochosos a partir de uma superfície inclinada, sem ocorrência de deformação apreciável da superfície (WP/WLI UNESCO, 1993). O movimento descendente pode ser feito por queda, ressalto ou rolamento.

A avaliação da susceptibilidade recorreu a métodos de modelação tridimensional, permitindo identificar i) a localização e características das principais áreas de génese do movimento de queda de blocos; ii) a trajectória e as áreas de projecção dos blocos deslocados.

\section{Caracterização da área}

A região de Alvaiázere apresenta unidades morfo-estruturais representativas da Orla Mesocenozóica e do Maciço Hespérico. No sector oriental da área de estudo aparecem representados os materiais do Maciço Hespérico, nomeadamente as Formações do Grupo de Cácemes (xistos, arenitos e siltitos), do Quartzito Armoricano e gnaisses tonalíticos do Proterozóico médio (SGP, 1992) em contacto a oeste com os materiais triásicos do Grupo Grés de Silves, Morfologicamente, dominam as serras e colinas de xistos, gnaisses e grauvaques, que servem de cabeceira às bacias dos rios Nabão e 
Zêzere em confronto, na depressão periférica ocidental, com os materiais sedimentares detríticos, e carbonatados, localmente evaporíticos.

No domínio da Orla salientam-se como elementos geomorfológicos mais relevantes: o vale do rio Nabão, a oeste, de orientação aproximada Norte-Sul, constituindo o limite natural do concelho de Alvaiázere com os concelhos de Ansião e Pombal e a Serra de Alvaiázere, com uma altura máxima de $618 \mathrm{~m}$, formada por calcários do Jurássico médio, nomeadamente envolvendo as Formações dos Calcários de Cabaços, Calcários de Sicó e Margas e calcários de S. Gião (SGP, 1992).

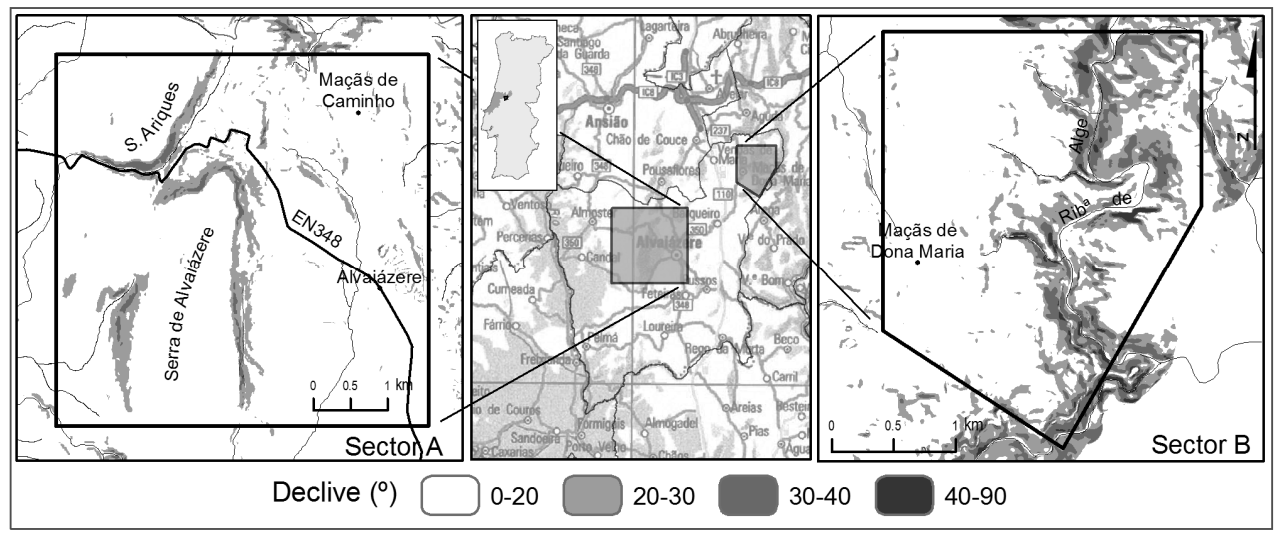

Figura 1. Enquadramento e classes de declive da área em estudo.

No conjunto, a orografia é marcada pelas colinas que conferem um aspecto ondulado à paisagem. As áreas de maior declive consistem nas vertentes da Serra de Alvaiázere e nas vertentes que definem as linhas de água do sector nascente do concelho, de onde se salientam os vales encaixados da Ribeira de Alge e seus afluentes (Figura 1). As áreas mais aplanadas consistem nos fundos de vale do Rio Nabão e da Ribeira da Murta e na Depressão de Alvaiázere.

A ocupação do solo dominante relaciona-se com a actividade agroflorestal, o que reflecte em parte os condicionamentos impostos pelas características pedológicas e morfológicas - solos na sua maioria pouco desenvolvidos e declives acentuados. As principais áreas urbanas são Alvaiázere, Pussos, Cabaços, Rego da Murta e Maçãs de D. Maria (Figura $1)$. 


\section{Metodologia}

\section{Extensão RockFall Analyst}

A modelação tri-dimensional do processo de queda de blocos foi realizada com recurso à aplicação RockFall Analyst (Centre for Risk Assessment for Geohazards Studies, University of Alberta ${ }^{\circledR}$ ), versão 1.1, que consiste numa extensão do programa de Sistemas de Informação Geográfica ArcGIS $\left(\mathrm{ESRI}^{\circledR}\right)$, A aplicação RockFall Analyst (RA) modela o processo físico da queda de blocos a partir de uma determinada origem e de acordo com as características da superfície e do bloco (Lan, 2007).

A aplicação deste modelo teve como dados de entrada:

- Modelo Digital de Terreno (MDT) com resolução espacial de $5 \mathrm{~m}$, elaborado a partir de cartografia vectorial produzida na escala 1:10.000 para a maior parte da área analisada, e escala 1:2000, para a área da vila de Alvaiázere e sua envolvente próxima.

- Áreas de alimentação (seeders, na linguagem da extensão RA), que identificam os locais onde tem início o movimento de queda do bloco. Neste trabalho optou-se por seguir um critério alargado, isto é, consideraram-se com potencial para gerar movimentos de queda as áreas com declive superior a $20^{\circ}$. Em cada polígono de declive superior a este valor foi definida uma malha de pontos espaçada $10 \mathrm{~m}$, longitudinal e latitudinalmente, os quais constituíram os pontos de início (seeder points) da modelação da queda de blocos.

- Propriedades da superfície (material properties na linguagem da extensão RA), que identificam as características físicas que condicionam o desencadeamento do movimento e condicionam o processo físico durante o mesmo. As propriedades da superfície consideradas são o ângulo de atrito $(\phi)$, o coeficiente de restituição normal $(\mathrm{Rn})$ e o coeficiente de restituição tangencial (Rt), cujos valores se apresentam nas Tabelas 1 e 2, adaptados ao contexto da área em análise a partir de dados constantes em Rocha (1981), Valejjo et al. (2002), Dorren \& Seijmonsbergen (2003) e Lan et al. (2007) recorrendo a cartografia geológica e de uso do solo.

Quanto aos coeficientes de restituição observa-se que quanto maior o declive e menor a rugosidade do uso do solo, maiores são os valores de restituição normal e tangencial porque aumenta também a favorabilidade para o início e subsequente movimento de queda. 
Tabela 1. Ângulo de atrito adoptado.

\begin{tabular}{lc}
\hline & $\phi$ \\
\hline Calcários de Sicó - J2O & 40 \\
\hline Calcários de Cabaços (Calcários de Casmilo) - J3 & 35 \\
\hline Formação do Quartzito Armoricano: seq. ordovícica indiferenciada - OQABS** & 35 \\
\hline Gnaisses tonalíticos - PEM ${ }^{*}$ & 30 \\
\hline Grés de Silves - TJ & 35 \\
\hline Margas e calcários de Quiaios e de São Gião - J1 & 35 \\
\hline Ortognaisses migmatíticos - gz1m & 35 \\
\hline Xistos, grauvaques, chertes negros, vulcanitos ácidos e xistos porfiroblásticos - & 30 \\
\hline PES & 35 \\
\hline
\end{tabular}

Para além destes dados de entrada são indicadas as condições iniciais do movimento, como a massa do bloco, a velocidade inicial, a velocidade a partir da qual se considera cessado o movimento, entre outros (Tabela 3 ).

Tabela 2. Coeficiente de restituição normal e tangencial adoptado.

\begin{tabular}{|c|c|c|c|c|c|c|c|c|}
\hline \multirow{3}{*}{ Uso do Solo } & \multicolumn{8}{|c|}{ Declive } \\
\hline & \multicolumn{2}{|c|}{$60-90^{\circ}$} & \multicolumn{2}{|c|}{$40-60^{\circ}$} & \multicolumn{2}{|c|}{$30-40^{\circ}$} & \multicolumn{2}{|c|}{$0-30^{\circ}$} \\
\hline & $\mathbf{R n}$ & $\mathbf{R t}$ & $\mathbf{R n}$ & $\mathbf{R t}$ & $\mathbf{R n}$ & $\mathbf{R t}$ & $\mathbf{R n}$ & $\mathbf{R t}$ \\
\hline Erva & 0,45 & 0,95 & 0,4 & 0,9 & 0,35 & 0,85 & 0,3 & 0,8 \\
\hline Agrícola & 0,45 & 0,9 & 0,4 & 0,85 & 0,35 & 0,8 & 0,3 & 0,75 \\
\hline Agrícola, abandonada / $1^{\mathrm{a}}$ fase & 0,45 & 0,9 & 0,4 & 0,85 & 0,35 & 0,8 & 0,3 & 0,75 \\
\hline Agrícola, abandonada / $2^{\mathrm{a}}$ fase & 0,45 & 0,85 & 0,4 & 0,8 & 0,35 & 0,75 & 0,3 & 0,7 \\
\hline Agrícola, abandonada / $3^{\mathrm{a}}$ fase & 0,45 & 0,85 & 0,4 & 0,8 & 0,35 & 0,75 & 0,3 & 0,7 \\
\hline Mato & 0,45 & 0,85 & 0,4 & 0,8 & 0,35 & 0,75 & 0,3 & 0,7 \\
\hline Agro-florestal, agrícola, activa & 0,4 & 0,8 & 0,35 & 0,75 & 0,3 & 0,7 & 0,28 & 0,65 \\
\hline $\begin{array}{l}\text { Agro-florestal, agrícola, abandonada / } 1^{\mathrm{a}} \\
\text { fase }\end{array}$ & 0,4 & 0,8 & 0,35 & 0,75 & 0,3 & 0,7 & 0,28 & 0,65 \\
\hline $\begin{array}{l}\text { Agro-florestal, agrícola, abandonada / } 2^{a} \\
\text { fase }\end{array}$ & 0,4 & 0,8 & 0,35 & 0,75 & 0,3 & 0,7 & 0,28 & 0,65 \\
\hline $\begin{array}{l}\text { Agro-florestal, agrícola, abandonada / } 3^{a} \\
\text { fase }\end{array}$ & 0,4 & 0,8 & 0,35 & 0,75 & 0,3 & 0,7 & 0,28 & 0,65 \\
\hline Agro-florestal, florestal, activa & 0,4 & 0,75 & 0,35 & 0,7 & 0,3 & 0,65 & 0,28 & 0,6 \\
\hline $\begin{array}{l}\text { Agro-florestal, florestal, abandonada / } 1^{a} \\
\text { fase }\end{array}$ & 0,35 & 0,75 & 0,3 & 0,7 & 0,28 & 0,65 & 0,25 & 0,6 \\
\hline
\end{tabular}


Tabela 2 (continuação)

\begin{tabular}{|c|c|c|c|c|c|c|c|c|}
\hline \multirow{3}{*}{ Uso do Solo } & \multicolumn{8}{|c|}{ Declive } \\
\hline & \multicolumn{2}{|c|}{$60-90^{\circ}$} & \multicolumn{2}{|c|}{$40-60^{\circ}$} & \multicolumn{2}{|c|}{$30-40^{\circ}$} & \multicolumn{2}{|c|}{$0-30^{\circ}$} \\
\hline & $\mathbf{R n}$ & $\mathbf{R t}$ & $\mathbf{R n}$ & $\mathbf{R t}$ & $\mathbf{R n}$ & $\mathbf{R t}$ & $\mathbf{R n}$ & Rt \\
\hline $\begin{array}{l}\text { Agro-florestal, agrícola, abandonada / } 3^{\mathrm{a}} \\
\text { fase }\end{array}$ & 0,4 & 0,8 & 0,35 & 0,75 & 0,3 & 0,7 & 0,28 & 0,65 \\
\hline Agro-florestal, florestal, activa & 0,4 & 0,75 & 0,35 & 0,7 & 0,3 & 0,65 & 0,28 & 0,6 \\
\hline $\begin{array}{l}\text { Agro-florestal, florestal, abandonada / } 1^{a} \\
\text { fase }\end{array}$ & 0,35 & 0,75 & 0,3 & 0,7 & 0,28 & 0,65 & 0,25 & 0,6 \\
\hline $\begin{array}{l}\text { Agro-florestal, florestal, abandonada / } 3^{a} \\
\text { fase }\end{array}$ & 0,35 & 0,75 & 0,3 & 0,7 & 0,28 & 0,65 & 0,25 & 0,6 \\
\hline Florestal, limpa & 0,35 & 0,75 & 0,3 & 0,7 & 0,28 & 0,65 & 0,25 & 0,6 \\
\hline Florestal, com erva & 0,35 & 0,7 & 0,3 & 0,65 & 0,28 & 0,6 & 0,25 & 0,55 \\
\hline Florestal, com mato & 0,35 & 0,7 & 0,3 & 0,65 & 0,28 & 0,6 & 0,25 & 0,55 \\
\hline Florestal, com outras & 0,35 & 0,7 & 0,3 & 0,65 & 0,28 & 0,6 & 0,25 & 0,55 \\
\hline Ocupação humana & 0,4 & 0,7 & 0,35 & 0,65 & 0,3 & 0,6 & 0,3 & 0,55 \\
\hline
\end{tabular}

Tabela 3. Propriedades de alimentação inicial (seeder properties) adoptadas.

\begin{tabular}{llll}
\hline Propriedades & & Unidade & \multicolumn{1}{l}{$\begin{array}{l}\text { Valores } \\
\text { assumidos }\end{array}$} \\
\hline Massa & & $\mathrm{kg}$ & 100 \\
\hline Distância entre blocos & & $\mathrm{m}$ & Malha 10 x 10 \\
\hline \multirow{2}{*}{ Velocidade inicial } & Horizontal & $\mathrm{m} / \mathrm{s}$ & 5 \\
\cline { 2 - 4 } & Vertical & $\mathrm{m} / \mathrm{s}$ & 2 \\
\hline Altura à superfície topográfica & $\mathrm{m}$ & 5 \\
\hline Configuração & $\mathrm{N}{ }^{\circ}$ de blocos no ponto de início & & 1 \\
\cline { 2 - 4 } estocástica & Intervalo angular & ${ }^{\circ}$ (graus) & 0 \\
\hline \multirow{3}{*}{$\begin{array}{l}\text { Limiar de translação e } \\
\text { paragem }\end{array}$} & Velocidade do movimento aéreo & $\mathrm{m} / \mathrm{s}$ & 1.5 \\
\cline { 2 - 4 } & Distância do movimento aéreo & $\mathrm{m}$ & 2 \\
\cline { 2 - 4 } & Ângulo do movimento aéreo & ${ }^{\circ}$ (graus) & 20 \\
\cline { 2 - 4 } & Velocidade mínima (paragem) & $\mathrm{m} / \mathrm{s}$ & 0.1 \\
\hline \multirow{2}{*}{ Opções } & Número de pontos em cada célula & & 2 \\
\cline { 2 - 4 } & $\begin{array}{l}\text { Tolerância para cálculo do } \\
\text { impacte }\end{array}$ & & 0.001 \\
\hline
\end{tabular}


Ponderação dos resultados provenientes da aplicação da extensão RockFall Analyst

Da modelação realizada resultaram dados em formato vectorial shape file relativos à trajectória do movimento - percurso e altura (aquando da ocorrência de salto/voo) - e à velocidade do movimento. No passo seguinte, estas três saídas do modelo foram convertidas para formato matricial (células de $10 \mathrm{~m} \times 10 \mathrm{~m}$ ) representando a frequência, expressa em $\mathrm{n} .^{\mathrm{o}}$ movimentos/célula, a altura $(\mathrm{m})$ e a energia $\left(\mathrm{kg} .(\mathrm{m} / \mathrm{s})^{2}\right)$.

Os valores matriciais foram posteriormente reclassificados em valores de 1 a 5 de acordo com o histograma de frequências - a frequência e a energia foram classificadas pelo método dos quantis; a altura foi classificada manualmente por pequenos ajustes à classificação em intervalos naturais. Estes scores parcelares foram ponderados de modo a se obter o score final de cada célula, de acordo com a seguinte expressão:

Score_final $=[$ Score_frequência $] * 0.5+[$ Score_altura $] * 0.2+[$ Score_energia $] * 0.3$

As classes de susceptibilidade foram estabelecidas a partir dos valores de score final, de acordo com os intervalos apresentados na Tabela 4.

Tabela 4. Classificação da susceptibilidade obtida por modelação tri-dimensional.

\begin{tabular}{ll}
\hline Valor final & Classe \\
\hline 0 & Não aplicável \\
\hline$] 0-1.66]$ & Moderada a baixa \\
\hline$] 1.66-3.33]$ & Elevada \\
\hline$] 3.33-5]$ & Muito elevada \\
\hline
\end{tabular}

\section{Estudos caso}

O método acima descrito foi aplicado a duas áreas de natureza geológica e geomorfológica distinta (Figura 1):

- Sector A: com uma área de 2514.83 hectares, abrange a Serra de Alvaiázere e parte da Serra de Ariques bem como as localidades de Alvaiázere e Maçãs de Caminho. Este sector insere-se na unidade morfoestrutural Orla Mesocenozóica Ocidental, onde a litologia predominante são margas e calcários das Formações de Margas e Calcários de S. Gião, Calcários de Sicó e materiais do Grupo Grés de Silves;

- Sector B: com uma área de 687.95 hectares, situa-se a nordeste de Alvaiázere, e distinguem-se duas áreas: uma a oriente que inclui o vale encaixado da Ribeira de Alge; e outra a ocidente, de morfologia mais suave e que inclui a localidade de Maçãs de Dona Maria. Este sector apresenta como litologia predominante gnaisses tonalíticos e quartzitos. 


\section{Resultados}

No sector A verifica-se cerca de $15 \%$ de área total com classe de susceptibilidade atribuída, dos quais 86 ha têm susceptibilidade "muito elevada" e 150 ha susceptibilidade "elevada" (Tabela 5). No sector B, a percentagem de área total classificada como "muito elevada" e "elevada" é muito superior à verificada no sector $\mathrm{A}$, sendo de 15.81 ha e 12.78 ha, respectivamente.

A cartografia de susceptibilidade produzida por modelação tridimensional nas áreas de alimentação consideradas (áreas de declive superior a $20^{\circ}$ ) evidenciam como áreas de susceptibilidade "muito elevada", fundamentalmente os sectores de vertente onde o declive é mais acentuado (Figura 2).

Tabela 5. Área por classe de susceptibilidade obtida por modelação nos sectores A e B.

\begin{tabular}{lllll}
\hline & \multicolumn{2}{c}{ Sector A } & \multicolumn{2}{c}{ Sector B } \\
\hline $\begin{array}{l}\text { Classe de } \\
\text { susceptibilidade }\end{array}$ & Área (ha) & Área (\%) & Área (ha) & Área (\%) \\
\hline Não aplicável & 2147 & 85.37 & 411 & 59.69 \\
\hline Moderada a baixa & 132 & 5.25 & 81 & 11.72 \\
\hline Elevada & 150 & 5.97 & 88 & 12.78 \\
\hline Muito elevada & 86 & 3.41 & 108 & 15.81 \\
\hline Total & 2515 & 100.00 & 688 & 100.00 \\
\hline
\end{tabular}

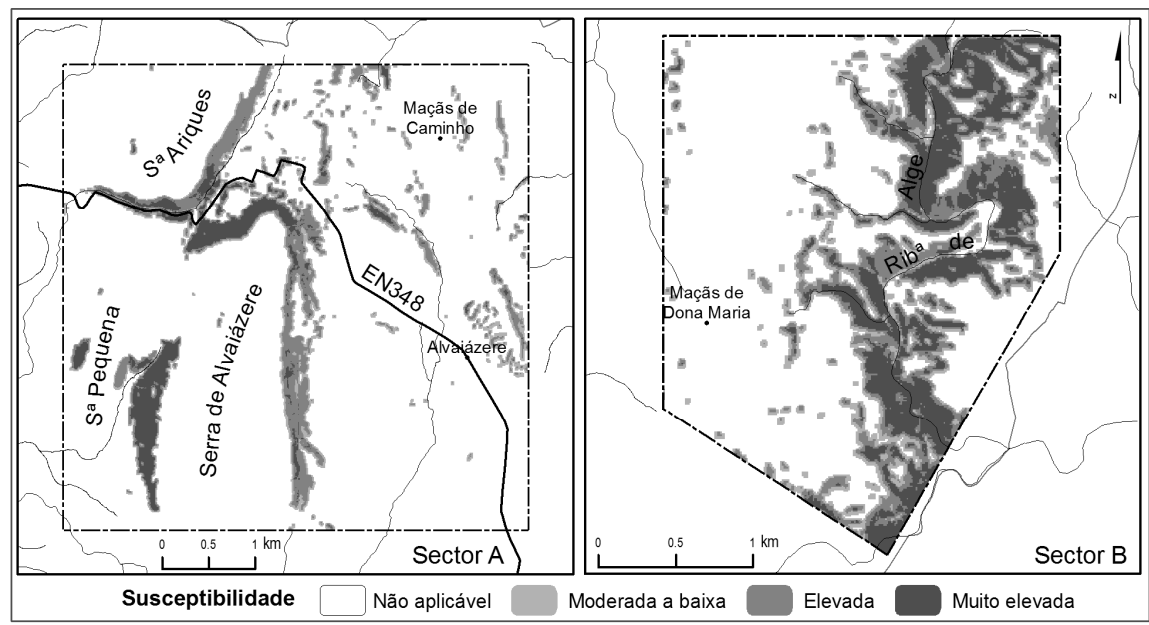

Figura 2. Susceptibilidade a queda de blocos por modelação tri-dimensional nos sectores $A$ e $B$. 
No sector A esta classe surge representada ao longo do Vale da Mata (canhão flúvio-cársico situado entre as serras de Ariques e Alvaiázere) afectando troços da EN 348 e nos flancos setentrional e ocidental da Serra de Alvaiázere. Com menor expressão, a classe "muito elevada" surge na Serra Pequena, no flanco oriental da Serra de Alvaiázere e em algumas vertentes envolventes à localidade de Maçãs de Caminho. No sector B, as classes de susceptibilidade "muito elevada" e "elevada" ocupam a quase totalidade das vertentes que definem o vale da Ribeira de Alge.

Para além da susceptibilidade a queda de blocos que resulta da ponderação dos outputs da extensão RA, é relevante analisar igualmente as áreas de projecção de blocos. Estes locais, quando correspondem a fundos de vale e a bases de vertente, não surgem na maior parte dos casos nas classes de susceptibilidade mais elevadas. Não obstante, a sua identificação é relevante pelo potencial de danos associado. A Figura 3 apresenta alguns exemplos de trajectórias modeladas no sector B (Maciço Antigo), onde se observa a forte correlação entre a distância percorrida pelo bloco e o declive das vertentes.

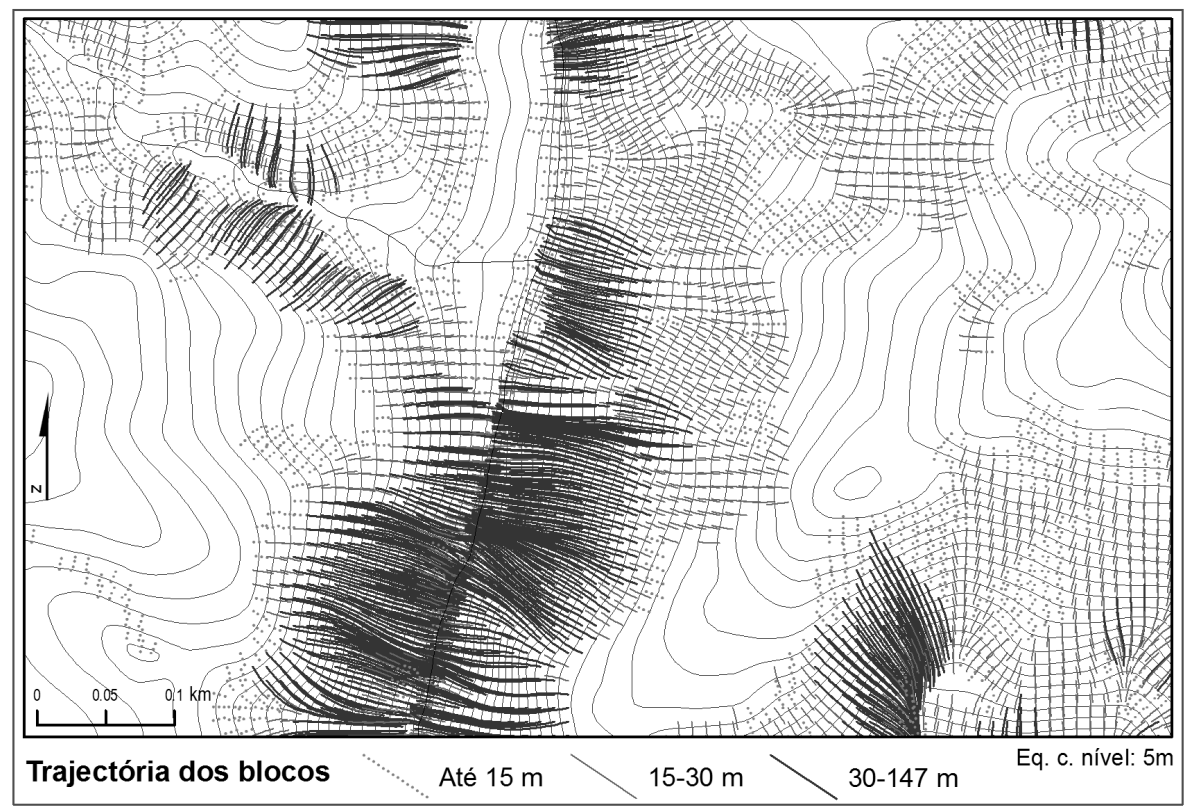

Figura 3. Exemplo de trajectórias calculadas pela extensão RockFall Analyst no sector $B$.

A comparação entre sectores - quanto à distância dos movimentos de queda de blocos - ressalta a diferença entre o número de blocos que 
percorre distâncias superiores a $30 \mathrm{~m}$ nos dois sectores (Tabela 6). Este valor é de $22.48 \%$ das trajectórias no sector B e apenas $0.99 \%$ no sector A. A justificação para estes números encontra-se fundamentalmente no facto de no sector B os declives serem em média superiores, mas parte da explicação pode residir também no facto de as vertentes no sector A possuírem um menor comprimento de vertente.

Tabela 6. Distância dos movimentos de quedas de bloco nos sectores A e B.

\begin{tabular}{lllll}
\hline & \multicolumn{2}{c}{ Sector A } & \multicolumn{2}{c}{ Sector B } \\
\hline Distância $(\mathbf{m})$ & Freq. abs. & $\mathbf{( \% )}$ & Freq. abs & Área (\%) \\
\hline$[0-15[$ & 9263 & 40.49 & 6426 & 34.42 \\
\hline$[15-30[$ & 13389 & 58.52 & 8046 & 43.10 \\
\hline$[30-147]$ & 227 & 0.99 & 4197 & 22.48 \\
\hline Total & 22879 & 100.00 & 18669 & 100.00 \\
\hline
\end{tabular}

\section{Notas finais}

A aplicação de modelação dos processos de queda de blocos recorrendo à extensão RA conduziu a dois tipos principais de resultados:

- identificação das principais áreas de alimentação, ou seja, aquelas onde o movimento de queda foi mais longo, não se ficando pelo o movimento proporcionado pelo impulso inicial definido pela velocidade horizontal e vertical.

- identificação das áreas de projecção, que surgem frequentemente com a classificação de susceptibilidade moderada a baixa devido à baixa frequência, energia e altura que as caracteriza.

$\mathrm{O}$ declive surge como principal factor condicionante, quer para o início da queda quer para a distância percorrida. A litologia, a partir da qual se extraíram os ângulo de atrito actua como um factor diferenciador da susceptibilidade de relevância inferior ao declive. Um exemplo disto é o que se observa entre os flancos oriental e ocidental da Serra Pequena. Nestes dois flancos o declive e o uso do solo são semelhantes e o único factor diferenciador é a litologia - no flanco oriental afloram Calcários de Sicó (ângulo de atrito $40^{\circ}$ ); no flanco ocidental afloram as Camadas de Cabaços (ângulo de atrito $35^{\circ}$ ). Esta diferença surge como explicação para o facto de a mancha de susceptibilidade "Muito elevada" ser superior no flanco ocidental, enquanto no flanco oriental, esta mesma classe de susceptibilidade se confina aos sectores de vertente com maior declive. 
O principal ponto fraco da metodologia aplicada reside no facto de se aplicarem factores de ponderação aos outputs da extensão RA por forma a definir a susceptibilidade, tendo verificado que os valores desta surgem subvalorizados nas áreas de projecção. Contudo, estas áreas são relevantes num contexto de gestão do risco dado o potencial de dano a elas associado, em particular afectando vias rodoviárias e habitações situadas para além das áreas de alimentação consideradas.

A aplicação da extensão RockFall Analyst pode constituir uma ferramenta complementar relativamente a outras metodologias de avaliação da susceptibilidade a queda de blocos, nomeadamente aos métodos heurísticos. Esta complementaridade torna-se evidente considerando a escassez de bases de dados com registos históricos de processos de queda de blocos.

\section{Bibliografia}

Dorren L.K.A., Seijmonsbergen A.C., (2003) - Comparison of three GIS-based models for predicting rockfall runout zones at a regional scale. Geomorphology; 56:49-64.

GSC (2002) - GSC Atlantic Rock Properties Database. Geological Survey of Canada/Dalhousie University. http://gsca.nrcan.gc.ca/pubprod/rockprop/search_e.php.

Lan H., Martin C.D., Lim C.H. (2007) - RockFall analyst: A GIS extension for three-dimensional and spatially distributed rockfall hazard modeling. Computers \& Geosciences; 33, 262-279.

Lan H., 2007. Manual for Rockfall Analyst. 3D Rockfall Modeling Extension to ArcGIS 9.x. $2^{\mathrm{a}}$ edição, Centre for Risk Assessment for Geohazards Studies, University of Alberta, 68 p.

Rocha M. (1981) - Mecânica das rochas. Laboratório Nacional de Engenharia Civil. 445 p.

SGP (1992) - Carta Geológica de Portugal (1:500.000). Serviços Geológicos de Portugal. Lisboa.

Vallejo, L., Ferrer, M., Ortuño, L., Oteo, C. (2002) - Ingeniería Geológica. Prentice Hall. Madrid, $717 \mathrm{p}$.

WP/WLI UNESCO (1993) - Multilingual landslide glossary. International Geotechnical Societies, Canadian Geotechnical Society (ed.), Richmond, BiTech Publishers Ltd.. 